\title{
BRAIN MRI ANATOMICAL AND ATTENTION AND BEHAVIOR DISORDERS WITH 22q11.2 DELETION SYNDROME
}

The brain anatomy of 39 children and adolescents with 22qDS (mean age 11 years; IQ 67) and 26 sibling controls (mean age 11 years; IQ 102) was compared using MRI and automated voxel-based morphometry, and behavioral differences were correlated with affected brain regions in a study at King's College, Institute of Psychiatry, London, UK; Royal College of Surgeons, Dublin, Ireland; and Academic Medical Center, Amsterdam, Holland. Individuals with 22qDS had a significant reduction in cerebellar grey matter, and white matter reductions in the frontal lobe, cerebellum and internal capsule; the volume of the occipital-parietal lobes was significantly reduced bilaterally; the right caudate nucleus and lateral ventricles were enlarged; and the prevalence of midline brain anomalies, such as cavum septum pellucidum, was increased. Significant positive correlations were found in 22qDS subjects between severity of (1) schizotypy symptoms and grey matter volume of temporo-occipital regions and the corpus striatum; and (2) emotional and (3) social behavioral problems and grey matter volume of frontostriatal regions. (Campbell LE, Daly E, Toal F, et al. Brain and behaviour in children with 22q11.2 deletion syndrome: a volumetric and voxel-based morphometry MRI study. Brain May 2006;129:1218-1228). (Respond: Linda Campbell, Centre for Mental Health Studies, James Fletcher Hospital, The University of Newcastle, Newcastle, NSW 2300, Australia).

COMMENT. Subjects with 22q deletion syndrome have changes in brain anatomy, especially white matter, basal ganglia and cerebellum, that correlate with behavioral disorders. Children with 22qDS have a higher prevalence of schizotypal traits, emotional symptoms, ADHD and social behavioral disorders. Frontostriatal regions found abnormal in $22 \mathrm{qDS}$ are implicated in attention and executive functions.

\section{PERINATAL DISORDERS}

\section{ETIOLOGY OF SPASTIC DIPLEGIA}

The clinical and etiologic profile of spastic diplegia was studied in a retrospective chart review of 54 patients diagnosed during a 12-year period at Montreal Children's Hospital, Quebec, Canada. Thirty-one (57.4\%) were preterm children, and 23 (42.6\%) term children. Initial concerns were gait abnormality in $18(33.3 \%)$, global developmental delay in $7(13 \%)$, motor delay in $6(11.1 \%)$, seizure in $3(5.6 \%)$, and hypotonia in $2(3.7 \%)$. ADHD was an associated comorbidity in $7(13 \%)$. The diagnosis was periventricular leukomalacia (PVL) in $24(44.4 \%)$ children; $26.1 \%$ were term and $58.1 \%$ preterm. The etiology was undetermined in $25(46.3 \%)$ children. Ischemic stroke occurred in $3(5.6 \%)$. PVL correlated with a birth weight of less than $2000 \mathrm{gm}$, history of neonatal resuscitation, and gestation less than 33 weeks. In term children, PVL was associated with perinatal difficulties, neonatal resuscitation, and a history of neonatal distress. (Tang-Wai R, Webster RI, Shevell MI. A clinical and etiologic profile of spastic diplegia. Pediatr Neurol March 2006;34:212-218). 